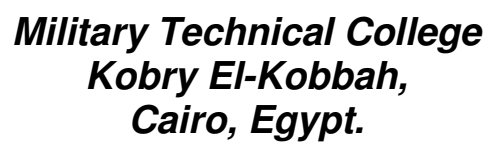

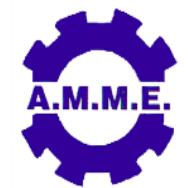

$18^{\text {th }}$ International Conference on Applied Mechanics and Mechanical Engineering.

\title{
FEATURE EXTRACTION ENHANCEMENT BASED ON PARAMETERLESS EMPIRICAL WAVELET TRANSFORM: APPLICATION TO BEARING FAULT DIAGNOSIS
}

\author{
H. H. El-Mongy*
}

\begin{abstract}
Rolling-element bearings are usually subject to faults that need prompt detection in order to prevent sudden failures. Many time-frequency analysis techniques have been used for the purpose of bearing fault detection and diagnosis. From these techniques, wavelets and empirical mode decomposition (EMD) stand out as the most widely applied methods in bearing fault diagnosis. Recently, a novel method named the parameterless empirical wavelet transform (PEWT) has been proposed to combine the wavelet formulation with the adaptability of the empirical mode decomposition. In this paper, the parameterless empirical wavelet transform (PEWT) is combined with envelope detection (ED) to present a new scheme named PEWT-ED for non-stationary signal analysis. The capabilities and limitations of the new method in bearing fault diagnosis are investigated using simulation and experiment. The results show that the new approach can effectively extract the bearing fault characteristics. The PEWT-ED is found to be a powerful tool in signal de-noising and enhancement for fault diagnosis purposes.
\end{abstract}

\section{KEYWORDS}

Rotating machinery, fault diagnosis, empirical wavelet transform, signal processing

* Assistant professor, Dept. of Mechanical Design, Faculty of Engineering-Mataria, Helwan University, Cairo, Egypt. 


\section{INTRODUCTION}

Rolling element bearing defects are generally the most common cause of failure in rotating machinery. Hence, close surveillance to detect bearing defects is essential to increase productivity, improve safety and reliability and prevent sudden failures of machinery. Conventional time-domain and Fourier-based analysis such as envelope detection (ED) have been widely applied for bearing fault diagnosis. These methods are based on the assumption of linearity and stationarity [1]. However, the presence of localized defects in bearings will modify the nature of system's vibration response and usually induces non-linearities and non-stationary events to emerge in the vibration signal [2]. Hence, using conventional methods may lead to incorrect diagnosis or may miss important information contained in a signal. Moreover, several studies indicated that some faults such as rotor cracks and rotor-stator rubbing are better diagnosed using non-stationary run up or coast down vibration response [3-8]. Therefore, advanced time-frequency signal analysis has become crucial part in fault diagnosis of rotating machinery. For instance, signal processing techniques can be utilized for signal decomposition, fault feature extraction, signal de-noising, feature classification, etc $[9,10]$.

Wavelet-based methods including continuous wavelet transform (CWT), discrete wavelet transform (DWT) and wavelet packet transform (WPT) have been successfully applied to detect various types of faults in rotating machinery such as bearing faults [11, 12], gear faults [13, 14], rotor cracks [15-17], rotor-stator rub [7, 18], etc. Excellent reviews on the use of wavelet analysis in fault diagnosis can be found in $[19,20]$. However, the results obtained from the wavelet transform is dependent upon the pre-specified analyzing wavelet, i.e. only signal features that match with the wavelet function will yield high coefficients.

Huang et al. [21] proposed the empirical mode decomposition method (EMD) as a self-adaptive technique that overcomes the drawback in the wavelet decomposition. EMD decomposes a signal into intrinsic mode functions (IMFs) which represent the natural oscillatory modes embedded in the signal. Nevertheless, EMD has its shortcomings; mode mixing, end effects and lack of theoretical basis [22]. Mode mixing means the assignment of the same frequency component to different IMFs. To overcome the mode mixing problem, the ensemble empirical mode decomposition (EEMD) was introduced [23, 24]. The EEMD makes use of noise assisted signal analysis concept to reduce mode mixing. Although EEMD has shown increased efficiency over the EMD in fault diagnosis applications but still remains some limitations; residual noise in the reconstructed signal and absence of selection criteria for the noise amplitude and number of ensemble [22]. Moreover, it is considered very expensive in terms of computation time and resources. Despite these drawbacks, EMD and EEMD have been successfully applied for fault diagnosis of various rotating machinery faults $[1,6,25-34]$.

Most recently, Gills [35] developed a new time-frequency method called empirical wavelet transform (EWT) which combines the solid theoretical basis of wavelets with the adaptability of EMD. In EWT, a wavelet filter bank is adaptively built based on segmentation of the spectrum of the processed signal. The EWT has been recently applied to decomposition of biomedical signals [36] and non-stationary signal analysis for gear and bearing fault detection as outlined hereafter. Cao et al. [37] applied the empirical wavelet transform to practical vibration signals of a wheel 
bearing of a train and found that it can be effective in bearing fault detection. It was noted that the spectrum segmentation step needs more study to enable adaptivity. Kedadouche et al. [38] used the concept of combined mode functions proposed by Gao et al. [33] . The empirical wavelet transform is applied to the combined mode functions to detect resonances and bearing defects even in noisy signals. Kedadouche et al. [39] compared the empirical wavelet transform and the empirical mode decomposition methods (EMD and EEMD) for bearing fault diagnosis. It was found that the EWT showed better results and lower computation time in comparison with EMD methods. Merainani et al. [28] combined the empirical wavelet transform with Hilbert transform for early diagnosis of gear tooth crack defect in both noisy and non-noisy environments. It was concluded that using EWT enhances the readability of the resulting time-frequency representation and enables early detection of incipient gear defects.

However, the EWT showed some drawbacks, mostly associated with the step of segmenting the signal spectrum into frequency bands. Too narrow frequency bands may cause overestimation of the number of extracted modes and accordingly unnecessary redundancy. Meanwhile, too wide frequency bands may result in poor extraction of useful features. Most recently, there are some attempts to improve the conventional EWT procedure. Hu et al. [40] used the envelope approach based on the order statistics filter to pick useful peaks from the spectrum before segmentation and the method was applied to noisy and non-stationary signals. In another recent study, Hu et al. [41] developed an adaptive and tacholess order analysis technique using the enhanced EWT to detect bearing faults in variable speed applications. Pan et al. [42] introduced a modified EWT (MEWT) method via data-driven adaptive Fourier spectrum segmentation that is suitable for mechanical fault diagnosis and applied it successfully to bearing fault diagnosis. Gills and Heal [43] presented an improvement of the segmentation procedure using scale space representation (SSR) that enables parameterless empirical wavelet transform (PEWT) procedure. Hence, it can be observed from the presented literature survey that EWT-based procedures show a promising potential in fault diagnosis of rotating machinery. But, the existing studies so far have concentrated on applying the conventional EWT to signals or developing modifications to the segmentation step. To the best of the author's knowledge, no research has been found that investigates the application of the PEWT to vibration signals for fault diagnosis purposes. The new technique needs more exploration and development and this has motivated the present study.

In this paper, the parameterless empirical wavelet transform (PEWT) is applied to vibration signals for the purpose of exploring its capabilities in bearing fault feature extraction. A fault diagnosis method based on PEWT combined with envelope detection (ED) and kurtosis statistical parameter is proposed to enhance feature extraction of bearing faults. The method is tested using a simulation signal of a bearing fault and real measured signals of defective bearings.

The rest of the paper is organized as follows: The theoretical background of the EWT and PEWT is given. Next, the proposed PEWT-ED combined approach is introduced. Then, a simulation example is presented to illustrate the effectiveness of the new PEWT-ED approach over the EWT in signal decomposition. Experimental signals of bearing faults are analyzed using the combined PEWT-ED approach. Finally, the conclusions of the study are summarized. 


\section{THEORETICAL BACKGROUND}

\section{Empirical Wavelet Transform}

The empirical wavelet transform is a new approach developed by Gills [35] to build adaptive wavelets. The modes of a signal are extracted by building a family of empirical wavelets that are adaptive to the analyzed signal. From the Fourier point of view, this process is equivalent to constructing a set of band-pass filters. The wavelets are adaptive because the filters' supports depend on the location of information in the spectrum of the analyzed signal. The empirical wavelet transform involves two steps: (1) segmentation of the Fourier spectrum to separate bands centered around a specific frequency and of compact support; (2) building the empirical wavelets and applying them to the analyzed signal. The segmented bands correspond to the constituent modes of the signal.

Consider a real valued signal $f(t)$ whose Fourier spectrum is denoted as $\hat{f}(\omega)$. The first step to segment a spectrum restricted from 0 to $\pi$ into $N$ segments is to detect the local maxima in the spectrum and sort them in descending order. Then, the boundaries $\omega_{n}$ of each segment is defined as the midpoint between two consecutive maxima. Each segment is denoted as $\Lambda_{n}=\left[\omega_{n-1}, \omega_{n}\right]$. Hence, it can be seen that $\bigcup_{n=1}^{N} \Lambda \mathrm{n}=[0, \pi]$. A transient phase of width $2 \tau_{n}$ is defined for each $\omega_{n}$, where $\tau_{n}$ is assumed to be proportional to $\omega_{n}\left(\tau_{n}=\gamma \omega_{n}\right)$ and $0<\gamma<1$. It can be chosen according to the following condition:

$$
\gamma<\min _{n}\left(\frac{\omega_{n+1}-\omega_{n}}{\omega_{n+1}+\omega_{n}}\right)
$$

The empirical wavelets are defined as bandpass filters on each segment $\Lambda_{\mathrm{n}}$. The basis wavelet function is chosen to be the Meyer wavelet. The empirical scaling function and the empirical wavelets are defined as follows:

$$
\begin{aligned}
& \varphi_{n}(\omega)= \begin{cases}1 & \text { if }|\omega| \leq \omega_{n}-\tau_{n} \\
\cos \left[\frac{\pi}{2} \beta\left(\frac{1}{2 \tau_{n}}\left(|\omega|-\omega_{n}+\tau_{n}\right)\right)\right] & \text { if } \omega_{n}-\tau_{n} \leq|\omega| \leq \omega_{n}+\tau_{n} \\
0 & \text { otherwise }\end{cases} \\
& \Psi_{n}(\omega)= \begin{cases}1 & \text { if } \omega_{n}+\tau_{n} \leq|\omega| \\
\cos \left[\frac{\pi}{2} \beta\left(\frac{1}{2 \tau_{n+1}}\left(|\omega|-\omega_{n+1}+\tau_{n+1}\right)\right)\right] & \text { if } \omega_{n+1}-\tau_{n+1} \leq|\omega| \\
\sin \left[\frac{\pi}{2} \beta\left(\frac{1}{2 \tau_{n}}\left(|\omega|-\omega_{n}+\tau_{n}\right)\right)\right] & \text { if } \omega_{n}-\tau_{n+1} \leq|\omega| \leq \tau_{n+1} \\
0 & \text { otherwise }\end{cases}
\end{aligned}
$$

The function $\beta(x)$ is an arbitrary function in $C^{k}([0,1])$ such that

$$
\beta(x)=\left\{\begin{array}{l}
0 \text { if } x \leq 0 \\
1 \text { if } x \geq 1
\end{array}, \text { and } \beta(x)+\beta(1-x)=1 \quad \forall x \in[0,1]\right.
$$


Many functions satisfy these properties, the standard example of this function is [40]:

$$
\beta(x)=x^{4}\left(35-84 x+70 x^{2}-20 x^{3}\right)
$$

Similar to the conventional wavelet transform, the empirical wavelet transform can be defined. The approximation coefficients are defined as the inner product of the signal and the scaling function $\varphi_{1}$ :

$$
W_{f}^{\varepsilon}(0, t)=\left\langle f, \varphi_{1}\right\rangle=\int f(\tau) \overline{\varphi_{1}(\tau-t)} d \tau=\operatorname{IFFT}\left(\hat{f}(\omega) \overline{\widehat{\varphi}_{1}(\omega)}\right)
$$

The detail coefficients are the inner products of the signal and the empirical wavelets $\psi_{n}$ :

$$
W_{f}^{\varepsilon}(n, t)=\left\langle f, \psi_{n}\right\rangle=\int f(\tau) \overline{\psi_{n}(\tau-t)} d \tau=\operatorname{IFFT}\left(\hat{f}(\omega) \overline{\hat{\psi}_{n}(\omega)}\right)
$$

Hence, the reconstructed signal can be obtained as:

$$
\begin{aligned}
f(t) & =W_{f}^{\varepsilon}(0, t) * \varphi_{1}(t)+\sum_{n=1}^{N} W_{f}^{\varepsilon}(n, t) * \psi_{n}(t) \\
& =\operatorname{IFFT}\left(\widehat{W}_{f}^{\varepsilon}(0, \omega) * \hat{\varphi}_{1}(\omega)+\sum_{n=1}^{N} \widehat{W}_{f}^{\varepsilon}(n, \omega) * \widehat{\psi}_{n}(\omega)\right.
\end{aligned}
$$

where $(*)$ represents the convolution. Hence, the signal empirical modes can be written as follows:

$$
\begin{aligned}
& f_{0}(t)=W_{f}^{\varepsilon}(0, t) * \varphi_{1}(t), \\
& f_{k}(t)=W_{f}^{\varepsilon}(k, t) * \psi_{k}(t)
\end{aligned}
$$

\section{Parameterless Empirical Wavelet Transform}

The adaptivity of the EWT decomposition depends greatly on the spectrum segmentation step outlined above. This procedure is usually effective for signals with well separated frequencies. However, for noisy and non-stationary signals, poor segmentation may occur. Some local maxima may be generated from noise and nonstationary events and on the other hand, meaningful maxima may be missed [40]. The performance of the EWT depends greatly on the number of bands, however, this number is not known a priori for real experimental signals and requires trial and error estimation. It can be estimated according to the purpose of signal decomposition and the type of fault to be diagnosed.

Another segmentation method has been proposed by Gills and Heal [43] based on scale-space representation (SSR). It has the advantage of being parameterless, i.e., it does not require giving the number of modes $N$ as an input to the EWT algorithm. In this method, the spectrum is seen as a histogram in which the occurrence of each frequency within a signal is counted. The delimiting boundaries are considered equivalent to finding local minima in the histogram. The meaningful modes are those 
having support delimited by two local minima which correspond to two long scalespace curves. Hence, the meaningful modes can be detected by finding a threshold $T$ such that scale-space curves of length larger than $T$ are the curves corresponding to minima delimiting modes' supports as shown in Fig. 1. This is considered a two class clustering problem on the set of scale space representations of the histogram/spectrum. It was concluded that although the parameterless scale-space procedure gives accurate estimate of the number of modes that are not known a priori, but too narrow frequency boundaries are usually detected for sophisticated signals. Hence, an overestimation of the number of modes may occur leading to unnecessary redundancy and spurious modes, i.e., modes lacking physical meaning.

\section{NEW PEWT-BASED PROCEDURE FOR BEARING FAULT DIAGNOSIS}

In this section, a new PEWT-based procedure for bearing fault diagnosis is presented. A bearing defect signal usually consists of impulses and modulation. Hence, a successful diagnosis of bearing defects is based upon extraction of impulses. Usually, the Fourier spectrum of raw vibration signal could not give obvious diagnostic information. Envelope demodulation technique has been an established benchmark method for bearing diagnostics for decades [10]. As the rolling element passes a local fault on the outer or inner race, or when a faulty rolling element strikes the inner or outer race, a shock pulse excites high frequency resonances of the bearing structure. These excited resonances are amplitude modulated on the bearing defect frequency that corresponds to the bearing fault, for example bearing outer race fault frequency (BPFO). In envelope detection (ED), a signal is band pass filtered in a high frequency band. Then, the impulses are amplified by the excited bearing structural resonances. Finally, the impulses are amplitude demodulated to form the envelope signal, whose spectrum contains valuable diagnostic information in terms of frequency as well as modulation.

The new approach presented here depends upon combining the PEWT decomposition and the envelope detection technique. The new procedure illustrated in Fig. 2 will be referred to as PEWT-ED throughout the paper. It is based on selecting the most informative PEWT components and summing them up to determine a meaningful reconstructed signal that characterizes the bearing fault. Then, envelope spectrum based on Hilbert transform of the meaningful reconstructed signal is obtained to show the bearing characteristic frequencies (BCF). The sensitive PEWT components are selected based upon calculating kurtosis for all the components and sorting the values to find the highest kurtosis modes. Kurtosis parameter is used as a statistical measure because of its sensitivity to the presence of spikes in a signal. It is well known that kurtosis value that is greater than 3 is an indicator of bearing damage [39]. Several previous studies used kurtosis as a statistical indicator for bearing fault diagnosis [13, 14, 39].

\section{SIMULATION EXAMPLE}

In this section, a simulated signal of a bearing defect is generated. The signal is carefully designed to contain bearing fault features; impulses and modulations. As shown in Fig. 3, the signal consists of a two-component harmonic signal with a train 
of modulated impulses and added noise. The sampling rate is taken as $12 \mathrm{kHz}$. The signal can be defined mathematically as [44]:

$$
x(t)=0.2 * \sin (2 \pi f t)+0.1 * \sin (2 \pi * 2 f t)+\sum_{i=1}^{3} e^{\left(-\beta_{i} t_{m}\right)} \sin \left(2 \pi i f_{i} t\right)+0.5 * n(t)
$$

where $f$ is the rotational frequency, $f_{i}$ is the resonance frequency of bearing and $\beta_{i}$ is a representative of damping of the impulse. Three resonance frequencies are considered in this example.

$t_{m}=\bmod \left(t, \frac{1}{f_{m}}\right)$

where $f_{m}$ is the modulating frequency and equals the defect characteristic frequency (BCF) and (mod) is a Matlab function which gives the modulus after division. A significant level of white Gaussian noise $n(t)$ is considered to simulate background noise present in real measured signals. Table 1 shows the values for the parameters used to construct this example.

Figure 4 shows the segmentation of the Fourier spectrum of the simulated signal using both conventional EWT and the proposed PEWT-ED. The dashed vertical lines represent the Fourier boundaries of the EWT method. For the case of conventional EWT, three different values for the number of modes are assumed; $N=10,15$ and 20. It can be seen that the chosen number of modes $N$ affects greatly the resulting spectrum segmentation. Increasing the number of modes refines the segmentation around peaks of the spectrum resulting in too fine boundaries. This enables the accurate detection of stationary harmonic components. But, till now there is no specific criteria for choosing the number of modes $N$, i.e. trial and error is used for estimating $N$. Meanwhile, the PEWT does not require prior information of the signal and the number of modes is automatically detected using scale-space representation. In comparison with conventional EWT segmentation, the PEWT boundaries are not too fine nor too wide. It can be noticed from Fig.4.d that 18 modes are detected by the PEWT. Figure 5 shows the signal decomposition using the conventional EWT $(N=20)$. Inspection of the constituent modes does not show the impulses contained in the signal. Meanwhile, the harmonic components are well separated in agreement with [40]. This implies that the conventional EWT is more suited to stationary signals rather that non-stationary signals.

Now, the PEWT-ED scheme is tested. The modes are extracted using the SSR segmented spectrum and the kurtosis parameter is calculated for all the modes. Then, the modes with the highest kurtosis are selected as meaningful modes and the rest of the modes are discarded. It is worth noting that choosing more than one mode is found to be more accurate than choosing the mode which exhibits the highest kurtosis. Hence, modes having kurtosis that is above a certain threshold are kept. The threshold of 0.7-0.9 of the kurtosis value of the original signal is found to be sufficient. Figure 6 shows the meaningful modes and the reconstructed signal using these modes only. Inspecting Fig. 6.a , the train of impulses can be easily detected. The envelope detection using Hilbert transform is then applied to the reconstructed signal. The resulting envelope spectrum is demonstrated in Fig. 7. The BCF at 100 $\mathrm{Hz}$ can be noticed in the envelope spectrum followed by its harmonics which is the classical symptom of bearing outer race fault for instance. 


\section{EXPERIMENTAL EXAMPLE}

In this section, real measured signals for defective bearings are used to test the effectiveness of the PEWT-based approach presented in this paper. The experimental signals are adopted from the Case Western Reserve University (CWRU) bearing data center available online at [45] for research purposes. The bearing data were collected from a 2-hp test rig. Vibration acceleration signals were acquired using a sampling frequency of $12 \mathrm{kHz}$ at 1730 revolution per minutes (RPM). For the signals used in this paper, faults were seeded on the inner race of the drive-end (DE) bearing (SKF 6205-2RS JEM) using electro-discharge machining $(E D M)$. The faults were generated in two severities; $0.18 \mathrm{~mm}$ and $0.53 \mathrm{~mm}$. The corresponding record numbers are 108DE and 212DE respectively. The bearing passing frequency for inner race fault (BPFI) equals $5.415^{\star} f_{r}$ and fundamental train frequency (FTF) equals $0.3983^{\star} f_{r}$ where $f_{r}$ is the rotational frequency in $\mathrm{Hz}$ [46]. To reduce the PEWT computation cost in terms of time and memory, a portion of the signal may be used instead of the whole signal. Ref. [39] suggested analyzing one shock pulse but, in the present work, the first 10,000 samples of both signals are employed. It is found that this choice is better from the diagnostic point of view especially in case of early stages of bearing fault in which impulses are nondistinguishable in signals.

Figure 8 shows the time records and corresponding segmented spectra using scale space representation. A series of impulses can be noticed in the time waveform for both records. The impulses are modulated over the shaft speed which is the rate at which the fault passes through the load zone. It is expected from the large number of frequency bands detected by the SSR segmentation algorithm that the resulting decomposition will contain some components that are not related to the bearing fault. Other components will show the bearing defect characteristics; impulses and modulations. Hence, the presented procedure PEWT-ED is followed here to isolate the useful components.

First, kurtosis value is calculated for all the PEWT components and the resulting values are then sorted in descending order to determine the set of modes with elevated kurtosis. The kurtosis values for PEWT components of both signals are plotted in Fig. 9. It may be noted that the kurtosis values for record 212 are generally higher than that for record 108 due to the increased fault severity. PEWT components having kurtosis values that are above a certain threshold are kept and the rest PEWT components are eliminated. For the two studied signals, threshold is taken as 3.3 and 5.6 respectively represented by the blue dashed lines in Fig. 9. Figure 10 shows these sensitive modes for both signals. Impulses modulated periodically at the shaft speed can be clearly seen in these components taking the characteristic pattern of a fish tail. Close inspection of the meaningful PEWT components shown in Fig. 10 reveals closely spaced impulses at (1/BPFI) and modulated over the rotational speed $f_{r}$. It is worth noting that these meaningful modes correspond to the region of excited bearing resonances in the range of $2000-4000$ $\mathrm{Hz}$. Next, these modes are summed together to form the meaningful reconstructed signal which manifests the bearing fault characteristics. Impacts and modulations can be clearly observed in the reconstructed signals for both severities as shown in Fig. 11. For lower severity (Fig. 11.a), the impacts are more obvious in comparison with the original signal shown in Fig. 8.a. 
Finally, the envelope spectra based on the Hilbert transform of the reconstructed signals are obtained and shown in Fig. 12. The bearing defect frequency that characterizes the inner race fault, i.e. BPFI can be seen clearly in both spectra along with its harmonics. Side bands at rotational frequency and double the rotational frequency appear around the BPFI component. Also, the rotational frequency $f_{r}$ and its harmonics can be observed. The dominance of $2 f_{r}$ in Fig. 12.a may suggest the presence of misalignment in the experimental test rig. Comparing the amplitudes of the defect frequency and its harmonics in Fig. 12.a and Fig. 12.b shows an increase which indicates a corresponding increase in the fault severity. Also, the increase in the spread and amplitudes of sidebands in Fig. 12.a indicates a corresponding increase in the impulsive modulation.

\section{CONCLUSION}

In this paper, a new procedure based upon parameterless empirical wavelet transform combined with envelope detection was presented and applied for bearing fault diagnosis. The kurtosis parameter is chosen as a selection criterion to isolate the meaningful PEWT components that are useful for bearing fault diagnosis. The simulation results showed that the PEWT can efficiently decompose a non-stationary signal that simulates bearing defect features. The conventional EWT necessitates specifying the number of components as an input to the EWT algorithm. Meanwhile, PEWT does not require prior information about the analyzed signal. Both simulation and experimental results showed that the proposed PEWT-ED procedure is effective in signal decomposition enhancement and feature extraction of the bearing fault characteristics. The PEWT helped in showing the impulses in the signal decomposition while the EWT failed to extract the impulses. The meaningful reconstructed signal attained by the PEWT-ED procedure and its envelope spectrum clearly demonstrated the features of the bearing fault. The influence of spurious modes and noise were effectively eliminated.

It is recommended to use the conventional EWT method for decomposing low-noise stationary signals. For noisy and impulsive signals such as bearing signature, it is suggested to use the PEWT-ED scheme rather than PEWT on its own due to the latter's overestimation of the number of components that yields spurious modes. More research is recommended to tackle improving spectrum segmentation algorithm. The effect of using wavelet functions other than Meyer wavelet needs investigation. For instance, Morlet wavelet is expected to give better results for localized fault detection as it has been proven suitable in impulse extraction for gear and bearing fault diagnosis in previous literature. In addition, the EWT applicability to diagnose other defects such as rotor faults needs more exploration.

\section{REFERENCES}

[1] L. Zhao, D. Huang, Y. Qin, Fault diagnosis for gearbox based on improved empirical mode decomposition, Shock and Vibration, 2015 (2015).

[2] Z. Feng, M. Liang, F. Chu, Recent advances in time-frequency analysis methods for machinery fault diagnosis: A review with application examples, Mechanical Systems and Signal Processing, 38 (2013) 165-205. 
[3] N.H. Chandra, A. Sekhar, Fault detection in rotor bearing systems using time frequency techniques, Mechanical Systems and Signal Processing, 72 (2016) 105-133.

[4] H.H. El-Mongy, Y.K. Younes, Vibration analysis of a multi-fault transient rotor passing through sub-critical resonances, Journal of Vibration and Control, (2017) 1077546317697828.

[5] D. Guo, Z. Peng, Vibration analysis of a cracked rotor using Hilbert-Huang transform, Mechanical Systems and Signal Processing, 21 (2007) 3030-3041.

[6] G. Gai, The processing of rotor startup signals based on empirical mode decomposition, Mechanical Systems and Signal Processing, 20 (2006) 222235.

[7] H. Ma, T. Yu, Q. Han, Y. Zhang, B. Wen, C. Xuelian, Time-frequency features of two types of coupled rub-impact faults in rotor systems, Journal of sound and vibration, 321 (2009) 1109-1128.

[8] H.H. El-Mongy, Y.K. Younes, Vibrational behaviour of a transient cracked rotor: Sub-critical and past-critical analyses, Engineering Research Journal 146 (2015) M69 - M92.

[9] A. Rai, S. Upadhyay, A review on signal processing techniques utilized in the fault diagnosis of rolling element bearings, Tribology International, 96 (2016) 289-306.

[10] R.B. Randall, J. Antoni, Rolling element bearing diagnostics-a tutorial, Mechanical systems and signal processing, 25 (2011) 485-520.

[11] J. Lin, L. Qu, Feature extraction based on Morlet wavelet and its application for mechanical fault diagnosis, Journal of sound and vibration, 234 (2000) 135-148.

[12] N. Nikolaou, I. Antoniadis, Rolling element bearing fault diagnosis using wavelet packets, Ndt \& E International, 35 (2002) 197-205.

[13] J. Lin, M. Zuo, Gearbox fault diagnosis using adaptive wavelet filter, Mechanical systems and signal processing, 17 (2003) 1259-1269.

[14] A. Ibrahim, S. Abd-elrahman, M. Zahran, H. El-Mongy, An experimental investigation for gear fault diagnosis using adaptive wavelet analysis of vibration signals, Bulletin of Faculty of Engineering, Faculty of Engineering, Menia University, 26 (2007).

[15] A. Sekhar, Crack detection through wavelet transform for a run-up rotor, Journal of Sound and Vibration, 259 (2003) 461-472.

[16] S. Adewusi, B. Al-Bedoor, Wavelet analysis of vibration signals of an overhang rotor with a propagating transverse crack, Journal of sound and vibration, 246 (2001) 777-793.

[17] J. Zou, J. Chen, Y. Pu, Wavelet time-frequency analysis of torsional vibrations in rotor system with a transverse crack, Computers \& structures, 82 (2004) 1181-1187.

[18] Z. Peng, F. Chu, W.T. Peter, Detection of the rubbing-caused impacts for rotorstator fault diagnosis using reassigned scalogram, Mechanical Systems and Signal Processing, 19 (2005) 391-409.

[19] Z. Peng, F. Chu, Application of the wavelet transform in machine condition monitoring and fault diagnostics: a review with bibliography, Mechanical systems and signal processing, 18 (2004) 199-221.

[20] R. Yan, R.X. Gao, X. Chen, Wavelets for fault diagnosis of rotary machines: A review with applications, Signal processing, 96 (2014) 1-15. 
[21] N.E. Huang, Z. Shen, S.R. Long, M.C. Wu, H.H. Shih, Q. Zheng, N.-C. Yen, C.C. Tung, H.H. Liu, The empirical mode decomposition and the Hilbert spectrum for nonlinear and non-stationary time series analysis, in: Proceedings of the Royal Society of London A: mathematical, physical and engineering sciences, The Royal Society, 1998, pp. 903-995.

[22] Y. Lei, J. Lin, Z. He, M.J. Zuo, A review on empirical mode decomposition in fault diagnosis of rotating machinery, Mechanical Systems and Signal Processing, 35 (2013) 108-126.

[23] Z. Wu, N.E. Huang, Ensemble empirical mode decomposition: a noise-assisted data analysis method, Advances in adaptive data analysis, 1 (2009) 1-41.

[24] P. Flandrin, G. Rilling, P. Goncalves, Empirical mode decomposition as a filter bank, IEEE signal processing letters, 11 (2004) 112-114.

[25] J. Lin, Q. Chen, Application of the EEMD method to multiple faults diagnosis of gearbox, in: Advanced Computer Control (ICACC), 2010 2nd International Conference on, IEEE, 2010, pp. 395-399.

[26] Y. Lei, Z. He, Y. Zi, Application of the EEMD method to rotor fault diagnosis of rotating machinery, Mechanical Systems and Signal Processing, 23 (2009) 1327-1338.

[27] G. Ibrahim, A. Albarbar, Comparison between Wigner-Ville distribution-and empirical mode decomposition vibration-based techniques for helical gearbox monitoring, Proceedings of the Institution of Mechanical Engineers, Part C: Journal of Mechanical Engineering Science, 225 (2011) 1833-1846.

[28] B. Merainani, D. Benazzouz, C. Rahmoune, Early detection of tooth crack damage in gearbox using empirical wavelet transform combined by Hilbert transform, Journal of Vibration and Control, 23 (2017) 1623-1634.

[29] B. Liu, S. Riemenschneider, Y. Xu, Gearbox fault diagnosis using empirical mode decomposition and Hilbert spectrum, Mechanical Systems and Signal Processing, 20 (2006) 718-734.

[30] L. Ming, L. Fucai, J. Beibei, B. Huiyu, L. Hongguang, M. Guang, Multi-fault diagnosis of rotor system based on differential-based empirical mode decomposition, Journal of Vibration and Control, 21 (2015) 1821-1837.

[31] R. Yan, R.X. Gao, Rotary machine health diagnosis based on empirical mode decomposition, Journal of Vibration and Acoustics, 130 (2008).

[32] V. Rai, A. Mohanty, Bearing fault diagnosis using FFT of intrinsic mode functions in Hilbert-Huang transform, Mechanical Systems and Signal Processing, 21 (2007) 2607-2615.

[33] Q. Gao, C. Duan, H. Fan, Q. Meng, Rotating machine fault diagnosis using empirical mode decomposition, Mechanical Systems and Signal Processing, 22 (2008) 1072-1081.

[34] C. Guo, M. Al-Shudeifat, J. Yan, L. Bergman, D. McFarland, E. Butcher, Application of empirical mode decomposition to a Jeffcott rotor with a breathing crack, Journal of Sound and Vibration, 332 (2013) 3881-3892.

[35] J. Gilles, Empirical wavelet transform, IEEE transactions on signal processing, 61 (2013) 3999-4010.

[36] A. Bhattacharyya, V. Gupta, R.B. Pachori, Automated identification of epileptic seizure EEG signals using empirical wavelet transform based Hilbert marginal spectrum, in: Digital Signal Processing (DSP), 2017 22nd International Conference on, IEEE, 2017, pp. 1-5.

[37] H. Cao, F. Fan, K. Zhou, Z. He, Wheel-bearing fault diagnosis of trains using empirical wavelet transform, Measurement, 82 (2016) 439-449. 
[38] M. Kedadouche, M. Thomas, A. Tahan, Empirical Mode Decomposition combined with Empirical Wavelets for extracting bearing frequencies in a noisy environment and early detection of defects, in: Advances in Condition Monitoring of Machinery in Non-Stationary Operations, Springer, 2016, pp. 151165.

[39] M. Kedadouche, M. Thomas, A. Tahan, A comparative study between Empirical Wavelet Transforms and Empirical Mode Decomposition Methods: Application to bearing defect diagnosis, Mechanical Systems and Signal Processing, 81 (2016) 88-107.

[40] Y. Hu, F. Li, H. Li, C. Liu, An enhanced empirical wavelet transform for noisy and non-stationary signal processing, Digital Signal Processing, 60 (2017) 220229.

[41] Y. Hu, X. Tu, F. Li, H. Li, G. Meng, An adaptive and tacholess order analysis method based on enhanced empirical wavelet transform for fault detection of bearings with varying speeds, Journal of Sound and Vibration, 409 (2017) 241255.

[42] J. Pan, J. Chen, Y. Zi, Y. Li, Z. He, Mono-component feature extraction for mechanical fault diagnosis using modified empirical wavelet transform via datadriven adaptive Fourier spectrum segment, Mechanical Systems and Signal Processing, 72 (2016) 160-183.

[43] J. Gilles, K. Heal, A parameterless scale-space approach to find meaningful modes in histograms-Application to image and spectrum segmentation, International Journal of Wavelets, Multiresolution and Information Processing, 12 (2014) 1450044.

[44] Y.-T. Sheen, A complex filter for vibration signal demodulation in bearing defect diagnosis, Journal of Sound and Vibration, 276 (2004) 105-119.

[45] Case Western Reserve University Bearing Data Center Website 〈http://csegroups.case.edu/bearingdatacenter/home) in 03/10/2017.

[46] W.A. Smith, R.B. Randall, Rolling element bearing diagnostics using the Case Western Reserve University data: A benchmark study, Mechanical Systems and Signal Processing, 64 (2015) 100-131. 


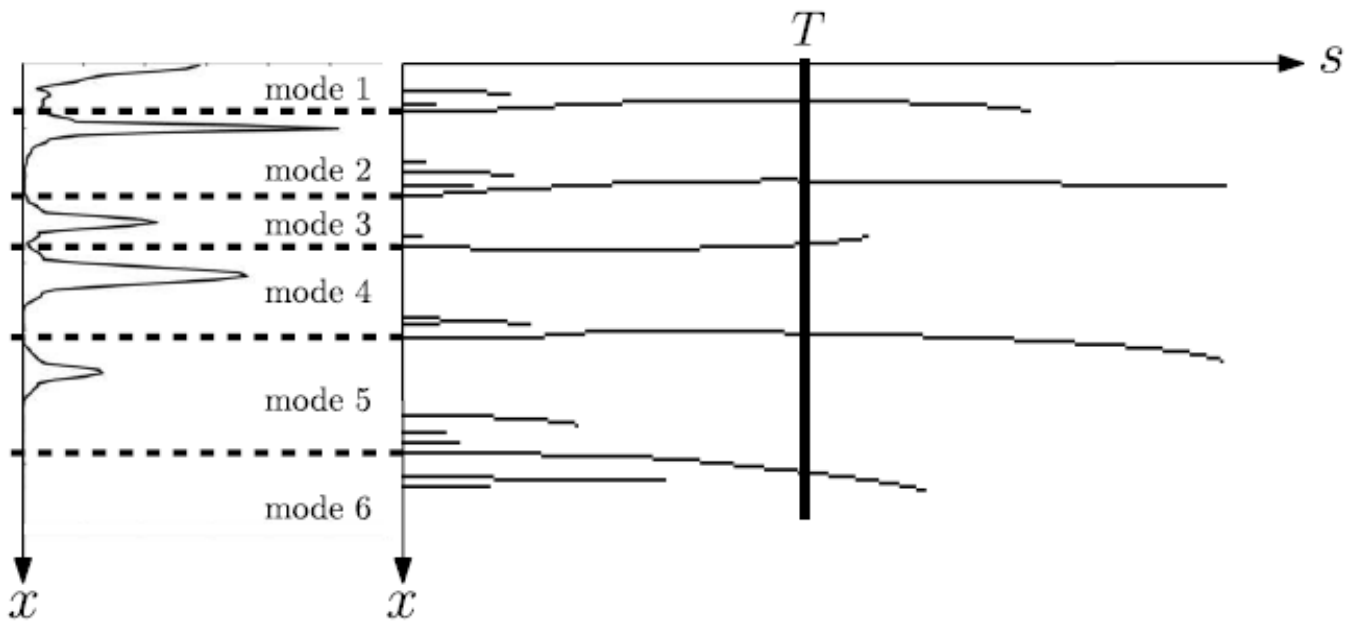

Fig.1. Mode detection using scale-space representation [43].

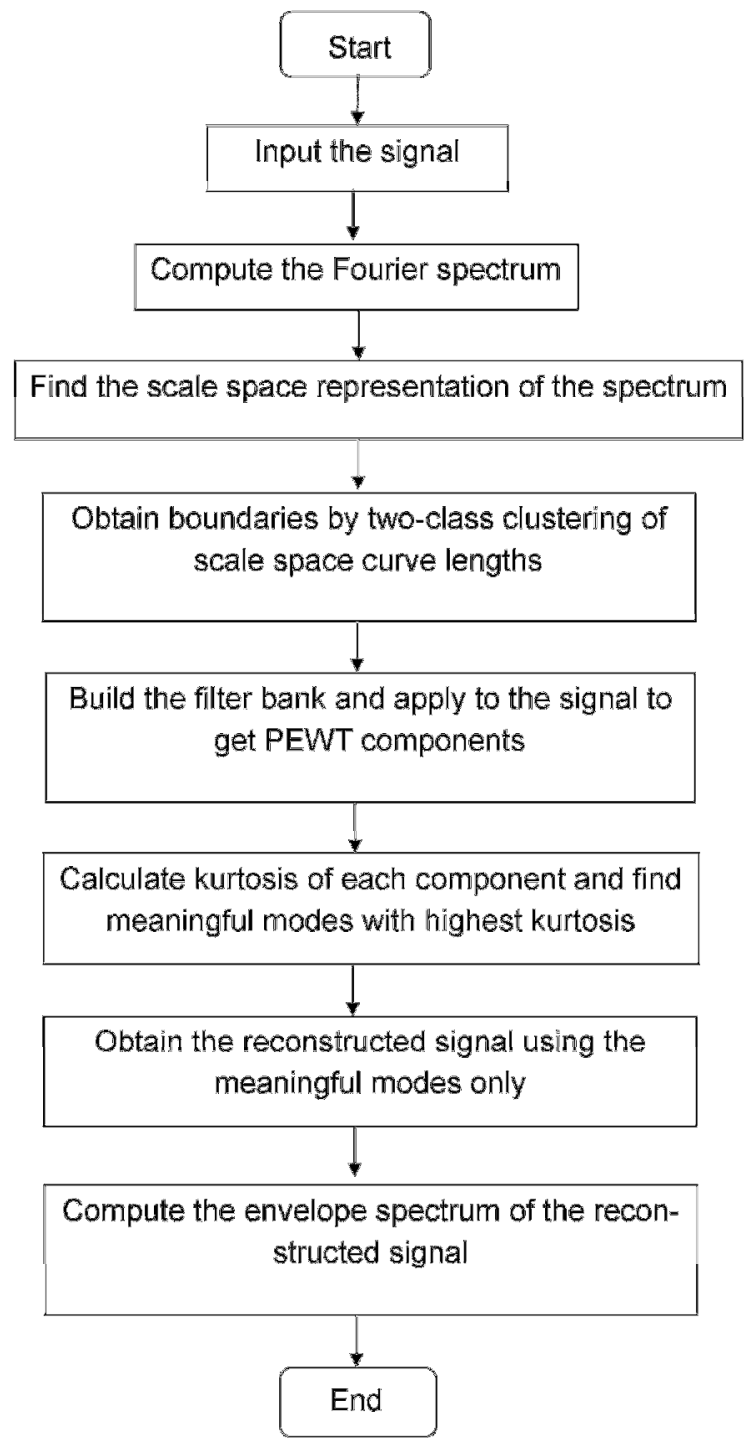

Fig. 2. Flow chart of the proposed PEWT-ED scheme. 
(a)

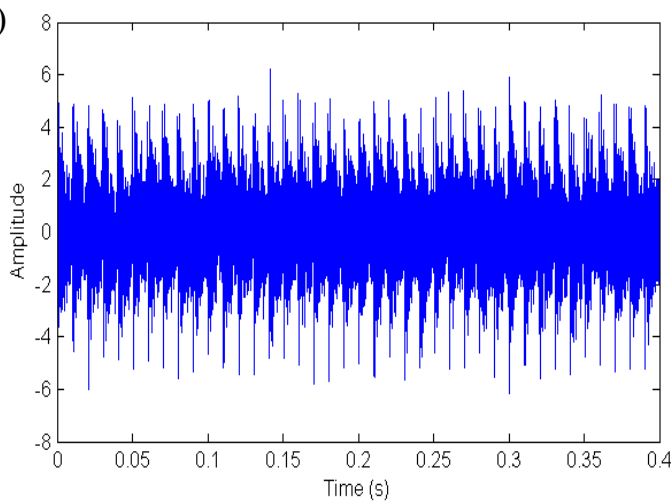

Fig. 3. Simulated bearing fault signal, (a) Time record, (b) Frequency spectrum. (b)

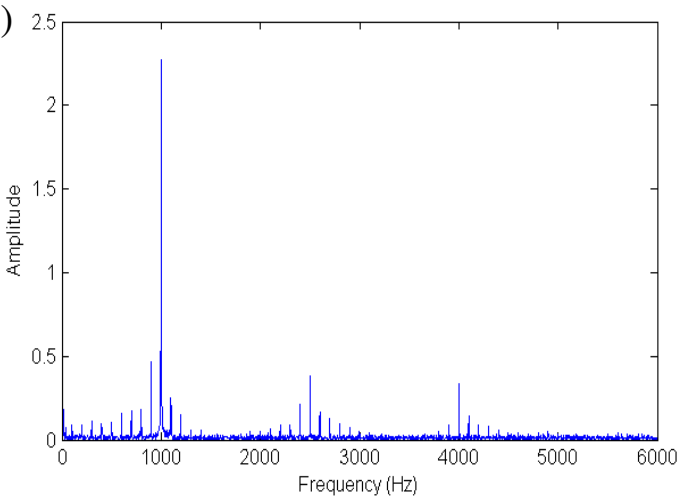

(a)

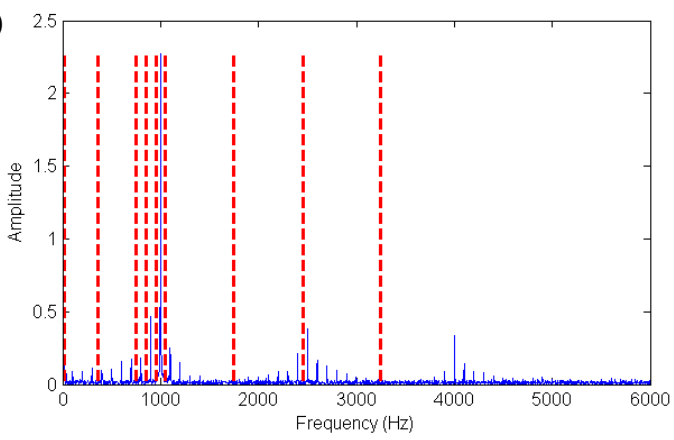

(c)

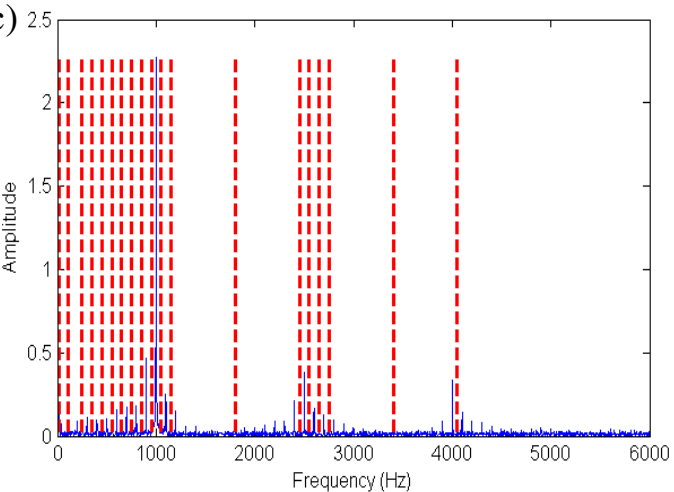

(b)

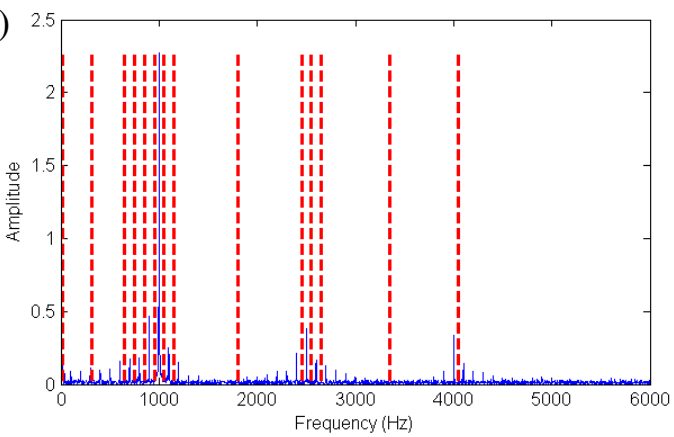

(d)

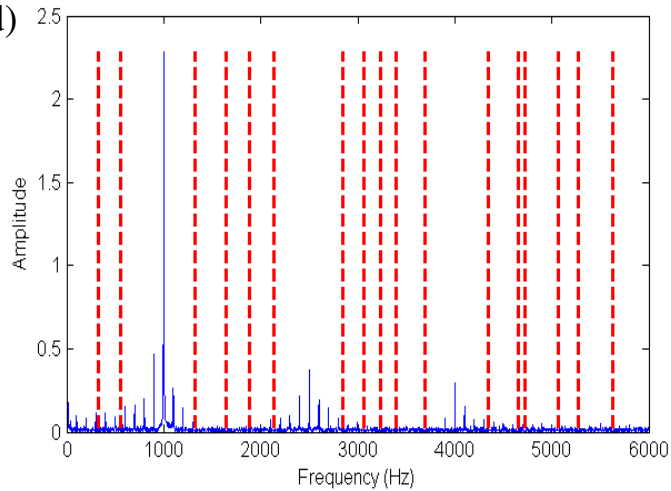

Fig. 4. Segmented frequency spectrum of simulated bearing defect signal, (a) Using conventional EWT ( $N=10)$, (b) Using conventional EWT $(N=15)$, (c) Using conventional EWT $(N=20)$, (d) Using scale space representation. 


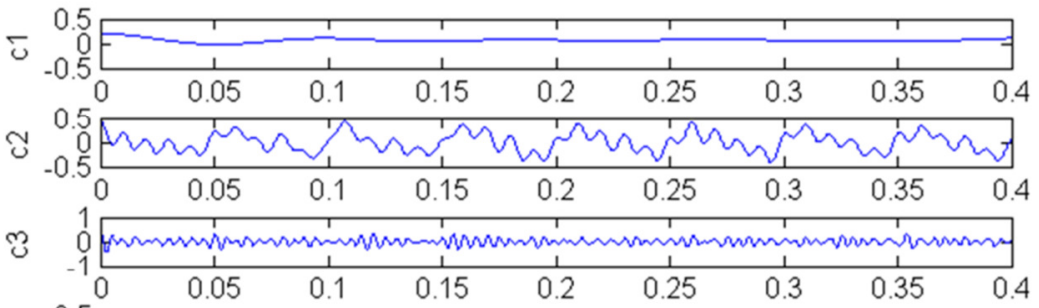

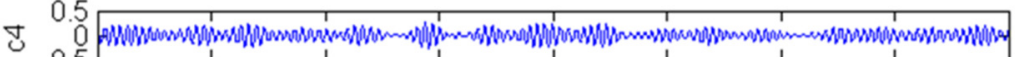

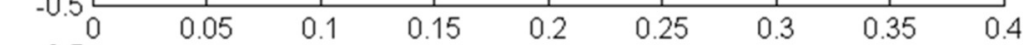

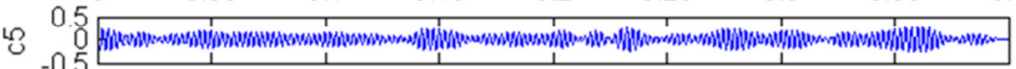

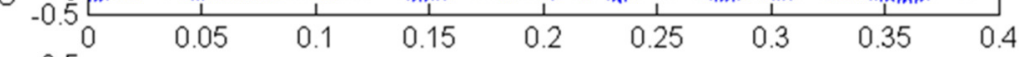

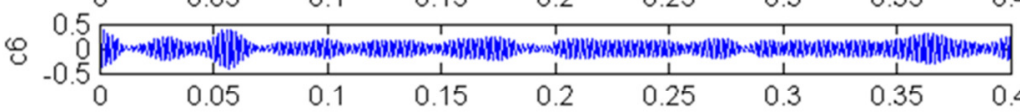

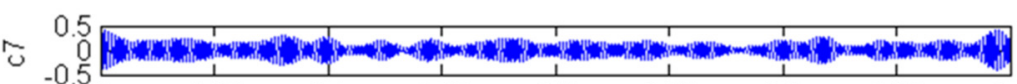

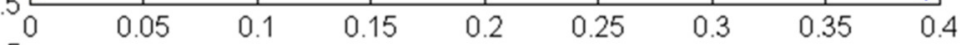

$\underset{0}{0} \begin{gathered}0.5 \\ 0.5\end{gathered}$

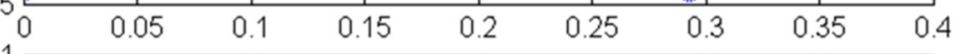

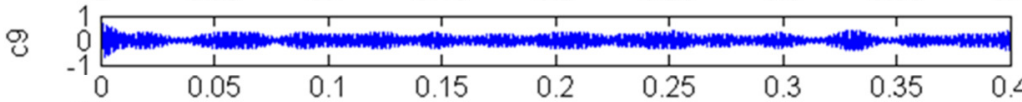
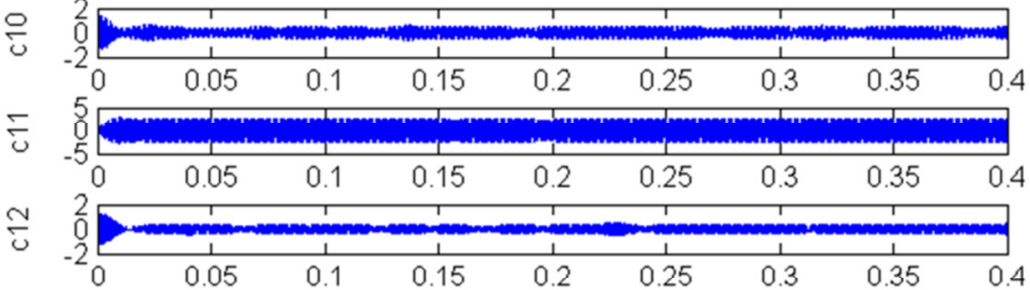

m ${ }_{0} \quad \begin{aligned} & 1 \\ & 0\end{aligned}$

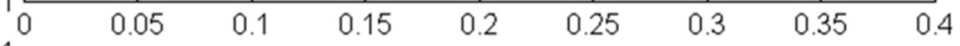

¿ $\quad \begin{aligned} & 0 \\ & 0\end{aligned}$

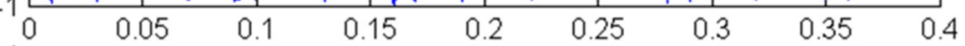
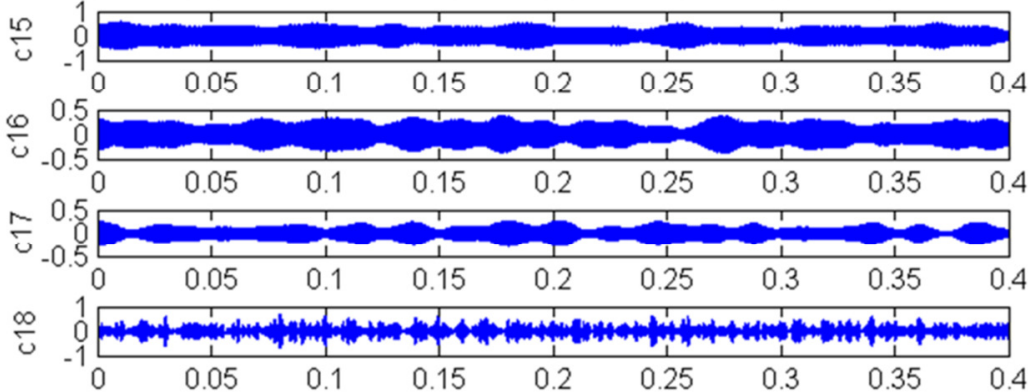

อ) $\int_{-1}^{1}$

今

Time (s)

Fig. 5. Conventional EWT decomposition of simulated bearing defect signal $(N=20)$. 

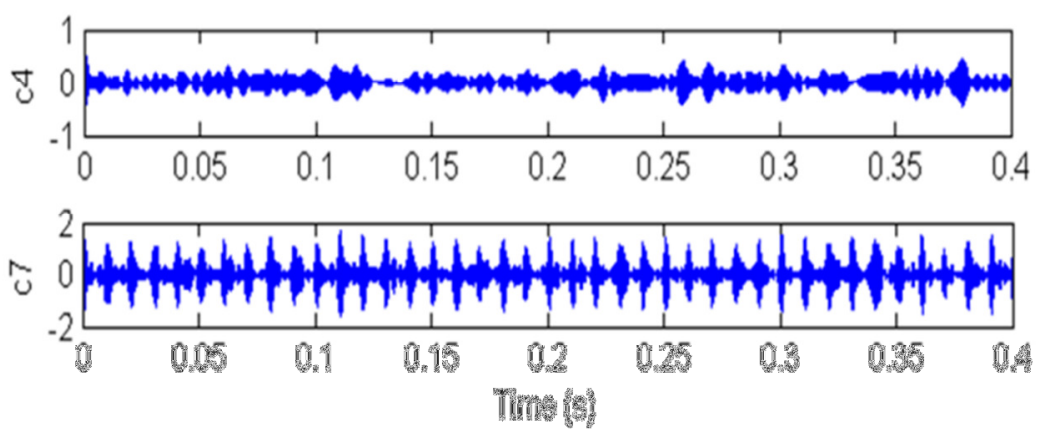

(a)

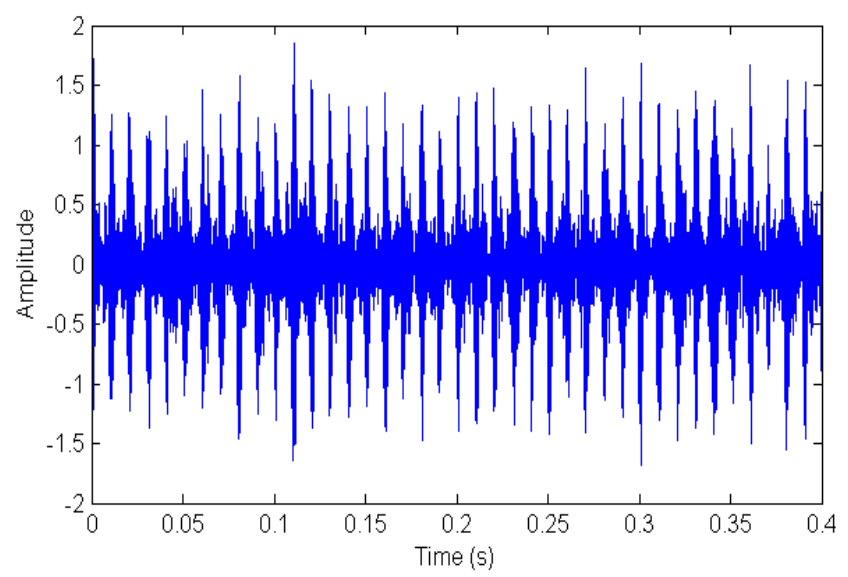

(b)

Fig. 6. PEWT-ED results for simulated bearing fault signal, (a) Meaningful components, (b) Meaningful reconstructed signal.

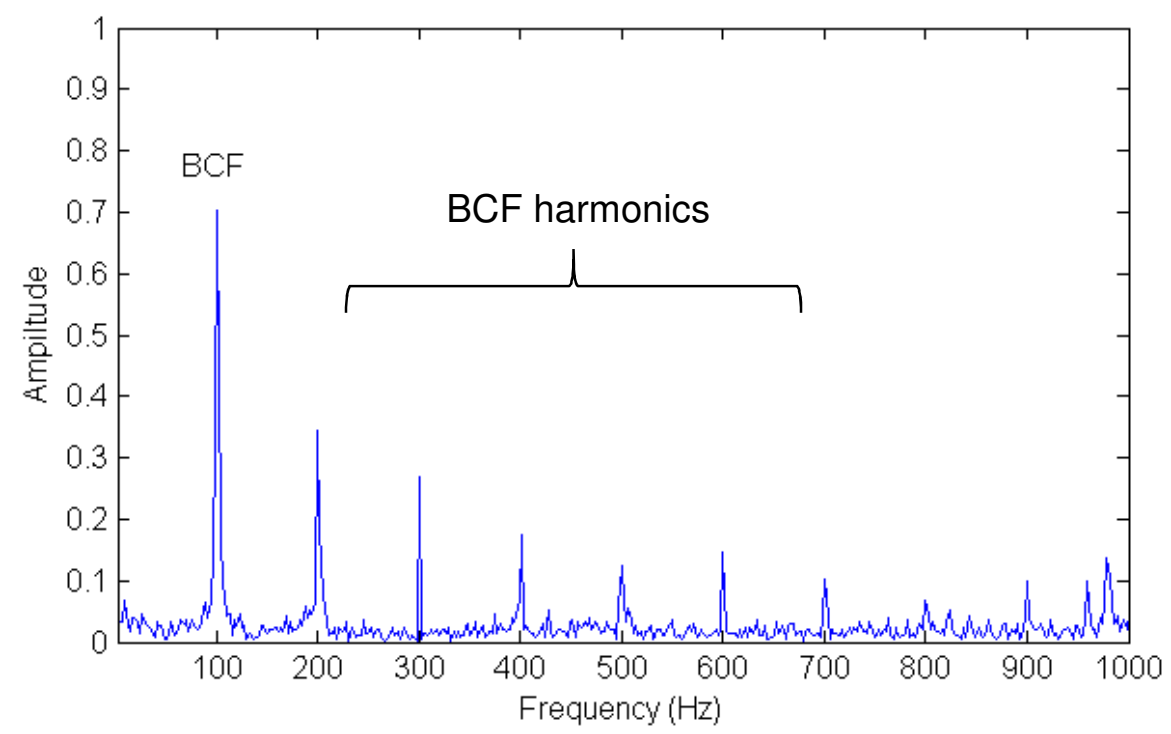

Fig. 7 Envelope spectrum of the meaningful reconstructed signal of simulated bearing fault signal. 
(i) Record 108DE

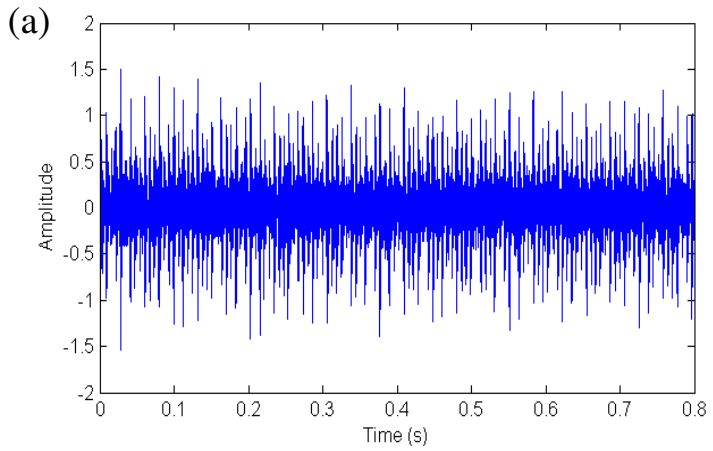

(b)

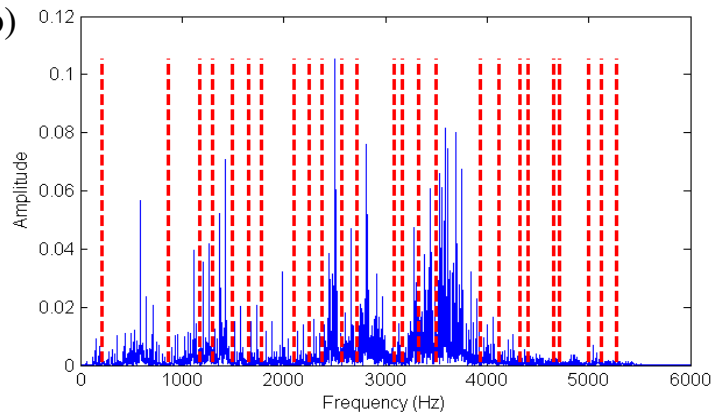

(ii) Record 212DE
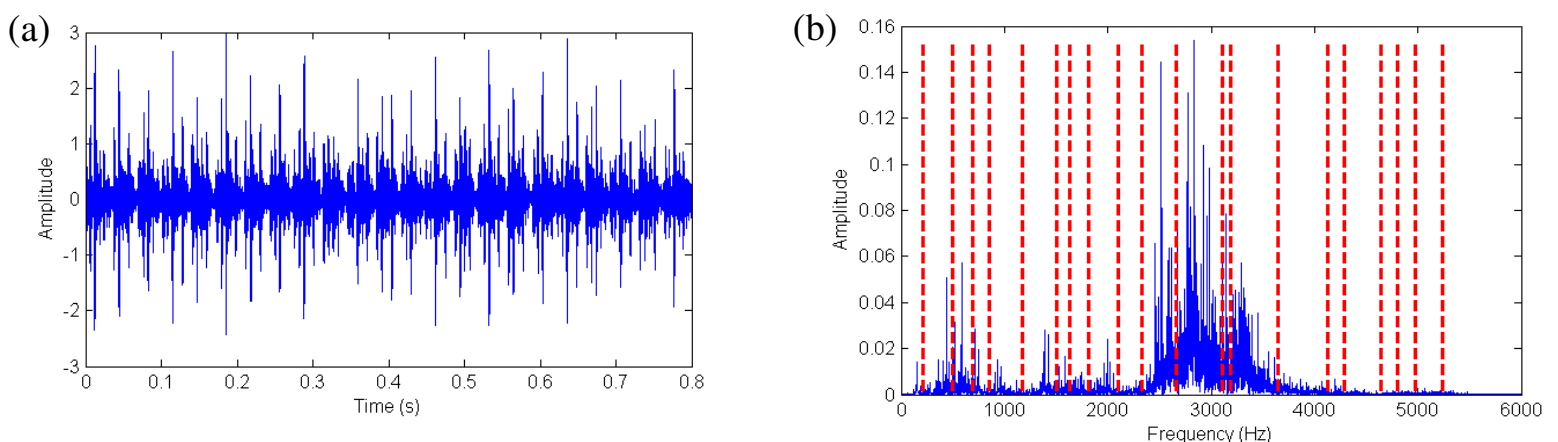

Fig. 8. Experimental bearing defect signals, (i) Record 108DE (0.18 $\mathrm{mm}$ inner race fault), (ii) Record 212DE (0.53 mm inner race fault), (a) Time record, (b) Segmented frequency spectrum using parameterless EWT.
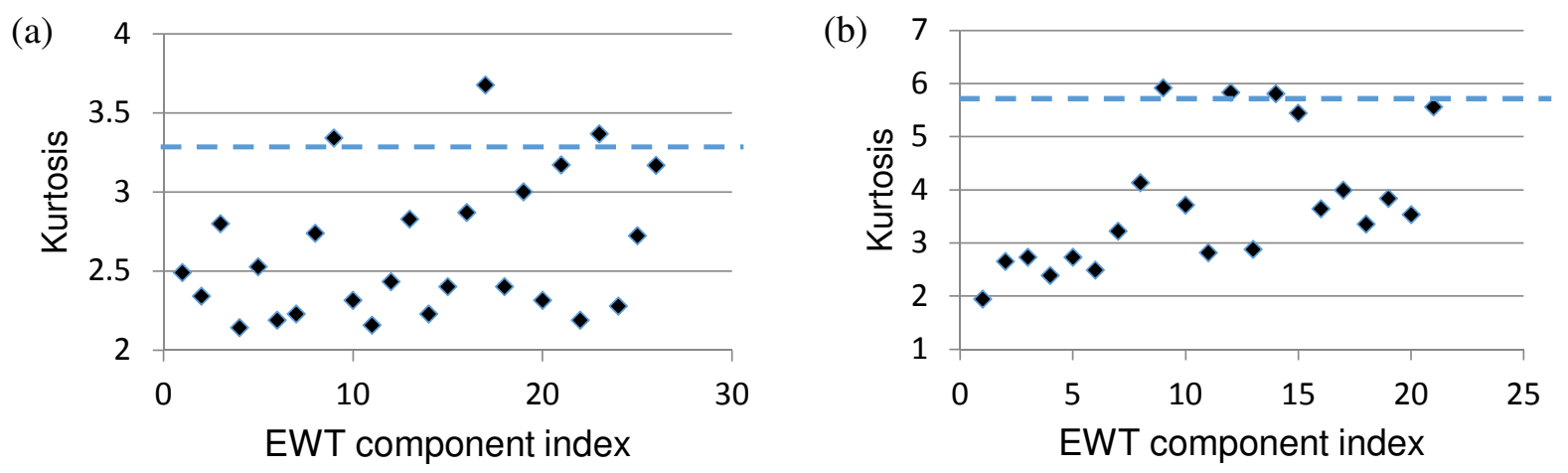

Fig. 9. Kurtosis values for PEWT components of signals (a) Record 108DE, (b) Record 212DE (The blue dashed line represents the threshold of kurtosis parameter). 
(a)
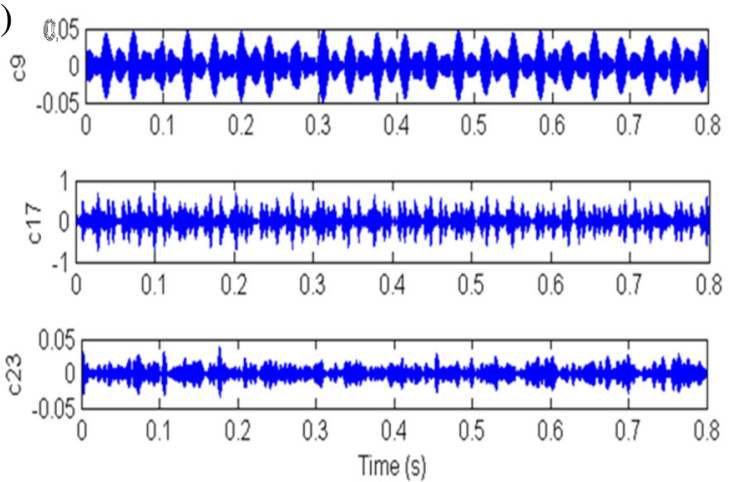

(b) 0.2
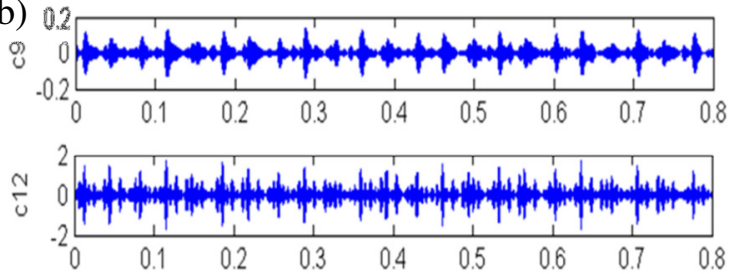

$\frac{\square}{\mathrm{U}}$

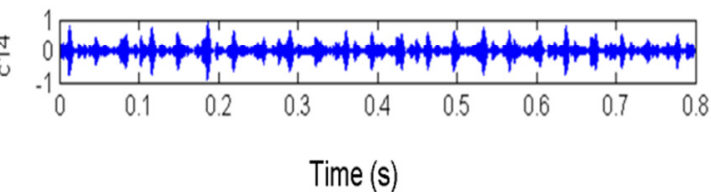

Fig. 10. PEWT components of highest kurtosis, (a) Record 108DE, (b) Record 212DE.

(a)

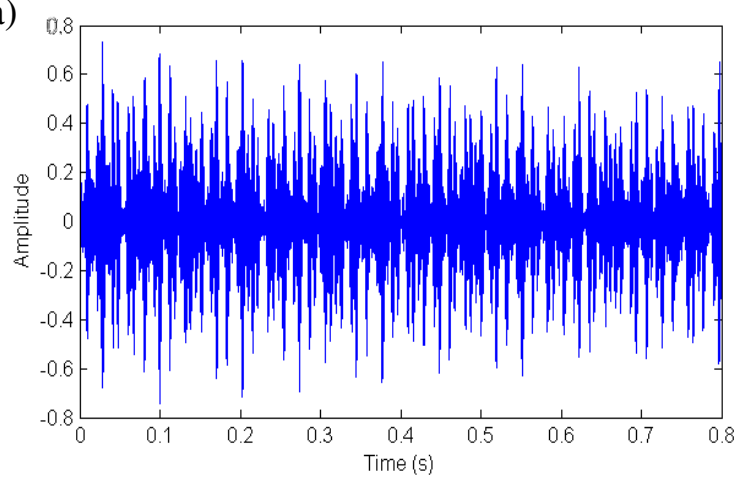

(b)

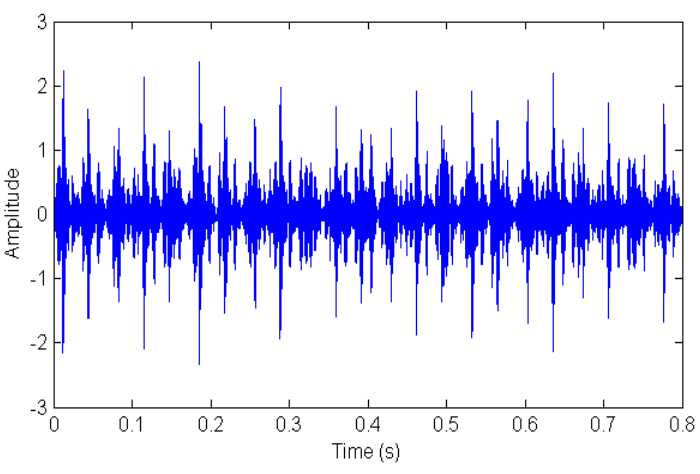

Fig. 11. Meaningful reconstructed signal, (a) Record 108DE, (b) Record 212DE.

(a)

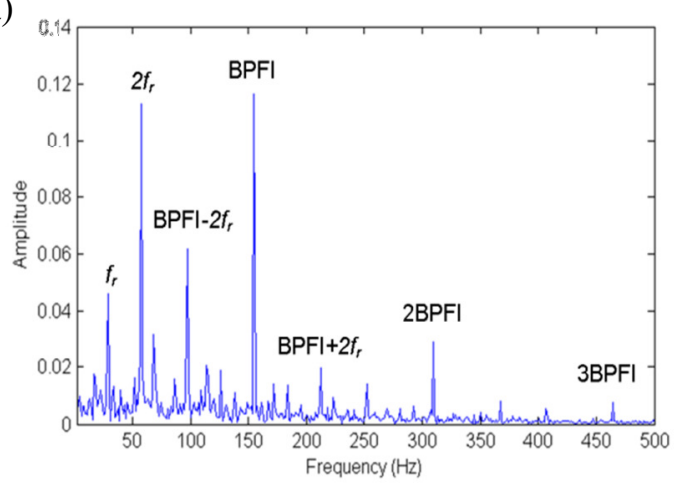

(b)

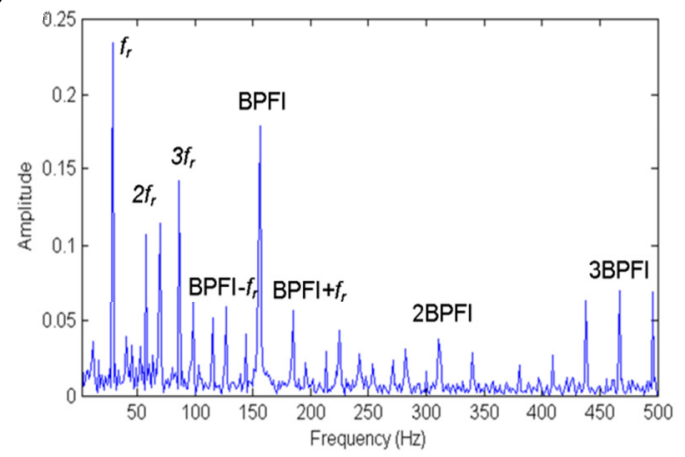

Fig. 12. Envelope spectra of meaningful reconstructed signals, (a) Record 108DE, (b) Record 212DE. 
Table 1. Simulated bearing defect signal parameters.

\begin{tabular}{|c|c|}
\hline Parameter & Value \\
\hline$f$ & $20 \mathrm{~Hz}$ \\
\hline$f_{m}$ & $100 \mathrm{~Hz}$ \\
\hline$f_{1}$ & $1000 \mathrm{~Hz}$ \\
\hline$f_{2}$ & $2500 \mathrm{~Hz}$ \\
\hline$f_{3}$ & $4000 \mathrm{~Hz}$ \\
\hline$\beta_{1}$ & 100 \\
\hline$\beta_{2}$ & 500 \\
\hline$\beta_{3}$ & 300 \\
\hline
\end{tabular}

